\title{
Instantiation of crystal plasticity simulations for micromechanical modelling with direct input from microstructural data collected at light sources
}

\author{
Reeju Pokharel and Ricardo A. Lebensohn ${ }^{1}$ \\ Los Alamos National Laboratory, Los Alamos, NM 87545, USA
}

\begin{abstract}
Novel non-destructive characterization techniques performed at light sources provide previously inaccessible 3-D mesoscopic information on the deformation of polycrystalline materials. One major difficulty for interpretation of these experiments through micromechanical modelling is the likelihood that processing and/or mounting the sample introduce residual stresses in the specimen. These stresses need to be incorporated into crystal plasticity formulations, for these models to operate directly from microstructural images and be predictive. To achieve this, the initial micromechanical state of each voxel needs to be specified. In this letter we present a method for incorporating grain-averaged residual stresses for instantiation of crystal plasticity simulations.
\end{abstract}

Keywords: microstructure, synchrotron radiation, micromechanical modeling, residual stresses, polycrystal plasticity.

${ }^{1}$ Corresponding author. Postal address: Materials Science and Technology Division, Los Alamos National Laboratory, MS G755, Los Alamos, NM 87845, USA. E-mail: lebenso@lanl.gov 


\section{1- Introduction}

Novel non-destructive techniques for microstructure characterization performed at light sources, such as high-energy X-ray diffraction microscopy (HEDM) [1], provide previously inaccessible 3-D in-situ information on the deformation of polycrystalline materials at the scale of the material's heterogeneity, i.e. the mesoscale. 3-D imaging is enabled by the collection of multiple diffraction patterns obtained by rotating the sample as it is quasi-statically deformed. Near-field (nf) HEDM allows characterization of crystal orientation fields in the form of voxelized microstructural images, while far-field (ff) HEDM provides local micromechanical information in the form of average stresses/elastic strains in the single crystal grains and the location of the centers of mass of the grains. One major difficulty of such techniques for interpretation of the deformation process through micromechanical modelling with direct input from the collected data is the likelihood that machining, or other type of processing (e.g. additive manufacturing), as well as handling, and/or mounting the sample introduce initial inter- and intragranular stresses in the specimen. These residual stresses (RS) need to be incorporated into full-field crystal plasticity $(\mathrm{CP})$ formulations, for these models to be able to operate directly from microstructural images and be predictive. To achieve this, the initial micromechanical state of each voxel in the image needs to be specified. However, if the initial grain average stresses measured by ff-HEDM were to be simplistically assigned to each voxel, this would lead to a non-equilibrated stress field in the specimen. In this letter we present a method for incorporating initial grain-averaged RS for instantiation of image-based CP simulations.

Concurrent and synergetic advances in material characterization and modelling techniques have opened vast opportunities to integrate increasingly sophisticated microstructural and micromechanical experiments and complex multiscale formulations, for simulating mechanical performance of polycrystalline materials (e.g. [2-3]). However, until very recently, the nature of the available experimental data was either statistical (e.g. X-ray or neutron diffraction), i.e. lacking space-resolved information on local fields, or destructive (e.g. Electron Back-Scattering Diffraction, EBSD), lacking information on microstructural and micromechanical evolution in the bulk of the material. These limitations are rapidly disappearing. Nowadays, powerful characterization techniques enabled by the availability of high energy radiation at light sources, are emerging to perform non-destructive space-resolved 3-D measurements. HEDM is one of such novel techniques [1, 4-6], providing spatially-resolved 3-D orientation fields [7-8] as well 
as grain-averaged elastic strain measurements for multiple states of a single sample subjected to external mechanical solicitation [9-10]. Such data has great potential for testing and calibrating full-field CP models [7, 11-12] and in turn these models can be instrumental to improve experimental data reduction. However, the likely introduction of RS due to fabrication and manipulation of HEDM samples determines the need for image-based $\mathrm{CP}$ formulations to be properly initialized, voxel by voxel, to assure predictive capability of these models when operating directly on HEDM images. The assignation of grain-averaged stresses measured by ffHEDM to each voxel is not a viable option, since this simplistic assumption would violate stress equilibrium in the specimen.

\section{2- Methods}

Our image-based CP formulation of choice is the well-established fast Fourier transform (FFT)based elasto-viscoplastic (EVPFFT) model [13] with the addition of an initial eigenstrain term [14] to reproduce the measured RS in the material and efficiently use HEDM input to predict micromechanical response of polycrystalline samples. However, despite the specific use here of FFT-based models, the proposed methodology is general, i.e. easily extendable and applicable to instantiate other popular full-field models, like CP Finite Elements (CPFE), from HEDM experimental data $[15,16]$.

While the reader is referred to [13] for a comprehensive description of the FFT-based model in the EVP regime, the main modification of the original EVPFFT formulation to consider "thermal" (eigenstrain) effects consists in the addition of such term in the local constitutive equation, which, at material point $\mathbf{x}$ and time $\mathrm{t}+\Delta \mathrm{t}$, is given by:

$$
\begin{aligned}
& \boldsymbol{\varepsilon}(\mathbf{x})=\boldsymbol{\varepsilon}^{\mathrm{e}}(\mathbf{x})+\boldsymbol{\varepsilon}^{\mathrm{p}}(\mathbf{x})=\mathbf{C}^{-1}(\mathbf{x}): \boldsymbol{\sigma}(\mathbf{x})+\boldsymbol{\varepsilon}^{\mathrm{p}, \mathrm{t}}(\mathbf{x})+\dot{\boldsymbol{\varepsilon}}^{\mathrm{p}}(\mathbf{x}, \boldsymbol{\sigma}) \Delta \mathrm{t} \\
& \boldsymbol{\varepsilon}(\mathbf{x})=\boldsymbol{\varepsilon}^{\mathrm{e}}(\mathbf{x})+\boldsymbol{\varepsilon}^{\mathrm{p}}(\mathbf{x})+\boldsymbol{\varepsilon}^{*}(\mathbf{x})=\mathbf{C}^{-1}(\mathbf{x}): \boldsymbol{\sigma}(\mathbf{x})+\boldsymbol{\varepsilon}^{\mathrm{p}, \mathrm{t}}(\mathbf{x})+\dot{\boldsymbol{\varepsilon}}^{\mathrm{p}}(\mathbf{x}, \boldsymbol{\sigma}) \Delta \mathrm{t}+\boldsymbol{\varepsilon}^{*}(\mathbf{x})
\end{aligned}
$$

Equation (1a) shows the original EVPFFT constitutive equation, relating the total local strain $\boldsymbol{\varepsilon}(\mathbf{x})$, given by the sum of the elastic $\varepsilon^{\mathrm{e}}(\mathbf{x})$ and plastic $\varepsilon^{\mathrm{p}}(\mathbf{x})$ strains, with the corresponding

stress field $\boldsymbol{\sigma}(\mathbf{x})$, where $\mathbf{C}^{-1}(\mathbf{x})$ is the local elastic compliance, $\boldsymbol{\varepsilon}^{\mathrm{p}, \mathrm{t}}(\mathbf{x})$ is the plastic strain at time t, and $\dot{\boldsymbol{\varepsilon}}^{\mathrm{p}}(\mathbf{x}, \boldsymbol{\sigma})$ is the plastic strain rate, constitutively related with the stress through a physically-based single crystal plasticity relation (see [13] for details). The addition and 
specification in Eq. (1b) of the eigenstrain field $\boldsymbol{\varepsilon}^{*}(\mathbf{x})$ extends the formulation to the thermoelasto-viscoplastic (TEVPFFT) regime, enabling input from ff-HEDM-type data.

In terms of 3-D datasets used in this work to assess the proposed method, for convenience, we performed a numerical experiment to generate and utilize synthetic data equivalent to a ffHEDM measurement, consisting of grain-averaged residual stresses/elastic strains. Such synthetic data was obtained applying the EVPFFT model to a numerically-generated polycrystalline unit cell, after loading-unloading the latter into the plastic regime. The proposed method, demonstrated and validated here for the aforementioned synthetic data, will be subsequently applied to actual HEDM data, e.g. ff-HEDM on smooth $\mathrm{Cu}$ samples measured at the European Synchrotron Radiation Facility (ESRF) [9]; or concurrent nf- and ff-HEDM on notched $\mathrm{Cu}$ samples measured at the Advanced Photon Source (APS) and to be reported elsewhere [17].

An initial approximation to convert observable data from ff-HEDM-type measurements, i.e. average elastic strains in the grains, into a meaningful instantiation of an image-based CP model (e.g. TEVPFFT) is given by what we call Eshelby's approximation, based on the known tensorial relation between a constant eigenstrain $\varepsilon_{\mathrm{ij}}^{*(\mathrm{~g})}$ in an ellipsoidal inclusion $(\mathrm{g})$ representing a grain, embedded in an infinite homogenous matrix, and the resulting uniform elastic strain $\varepsilon_{\mathrm{ij}}^{\mathrm{e}(\mathrm{g})}$ inside the inclusion, given by:

$\varepsilon_{\mathrm{ij}}^{*(\mathrm{~g})}=(\mathrm{S}-\mathrm{I})_{\mathrm{ijkl}}^{-1} \varepsilon_{\mathrm{kl}}^{\mathrm{e}(\mathrm{g})}$

where $\mathrm{S}$ is the fourth-rank Eshelby tensor, a known function (e.g. [18]) of the inclusion shape and the elastic stiffness of the material. In our calculations, we adopted the isotropic elastic constants $\mu=48.2 \mathrm{GPa}$ and $\nu=0.343$ corresponding to an elastic self-consistent approximation [19] for a $\mathrm{Cu}$ polycrystal with random texture and single crystal elastic constants $\mathrm{C}_{11}=68.4$, $\mathrm{C}_{12}=121.4, \mathrm{C}_{44}=75.4 \mathrm{GPa}$. Adopting the uniform elastic strain in Eq. (2) to be the measured average in each grain, i.e. $\varepsilon_{\mathrm{kl}}^{\mathrm{e}(\mathrm{g})}=\left\langle\varepsilon_{\mathrm{kl}}^{\mathrm{e}}\right\rangle^{(\mathrm{g})}$, the uniform eigenstrain calculated by means of the above expression can be used in a thermo-elastic FFT-based (TEFFT) calculation, to give micromechanical fields that fulfill stress equilibrium and strain compatibility and are consistent with the adopted eigenstrains in the grains. The resulting elastic strain field can then be averaged 
in each grain and these grain averages be compared with the corresponding measurements. As it will be shown in next section, the above described Eshelby's approximation leads to an imperfect correlation between synthetic (viz. "experimental") and predicted grain-averaged RS, and therefore needs to be improved. For this, we propose following modification to Eshelby's approximation (Eq. 2):

$\varepsilon_{\mathrm{ij}}^{*(\mathrm{~g})}=\beta_{\mathrm{ij}}(\mathrm{S}-\mathrm{I})_{\mathrm{ijkl}}^{-1} \varepsilon_{\mathrm{kl}}^{\mathrm{e}(\mathrm{g})} \quad($ no sum over $\mathrm{ij})$

where $\beta_{\mathrm{ij}}$ is a symmetric matrix of six independent correction factors (note that the strain tensors involved in Eq. (3) are themselves symmetric) that need to be adjusted for an optimal correlation between the instantiated and measured grain-averaged residual stresses and elastic strains. Results of the application of Eshelby's approximation, and the proposed methodology for its subsequent refinement based on the determination of the above correction factors $\beta_{\mathrm{ij}}$ are presented next.

\section{3- Results}

Figure 1 schematically illustrates the workflow implemented to assess Eshelby's approximation (Eq. 2) and its refinement using the correction factors $\beta_{\mathrm{ij}}$ (Eq. 3). A synthetic orientation field generated by Voronoi tessellation, representing a cylindrical polycrystalline sample with 14592 grains (\#1 in the figure), is used as input. Note that specific sample geometries (smooth cylindrical specimen in this case) can be handled using FFT-based methods (which operate on periodic unit cells) using appropriate buffer zones ("gas phase" with zero stiffness) that allow inscribing the cylinder in a prismatic unit cell to mechanically disconnect the original unit cell from its periodic repetitions along the lateral directions of the cylinder. This synthetic microstructure can eventually be replaced by the actual orientation field of a polycrystalline sample measured, e.g. by nf-HEDM, or obtained from ff-HEDM data with additional tessellation based on the centers of mass of the grains [20]. Next, a EVPFFT loading-unloading simulation up to $0.25 \%$ strain (\#2), using the $\mathrm{Cu}$ single crystal elastic constants given above and typical plastic constitutive parameters for $\mathrm{Cu}$ [17], was performed to obtain a synthetic residual elastic strain field (\#3). Taking grain averages of the latter (\#4), a set of grain-averaged residual elastic strains was obtained (\#5). This synthetic data, generated in steps \#2-\#5, can eventually be replaced by an actual ff-HEDM measurement. Processing the resulting grain-averaged residual 
elastic strains, a piecewise-constant eigenstrain field was calculated using Eshelby's approximation (\#6-\#7), and in turn used as input of a TEFFT calculation to obtain equilibrated stress and corresponding elastic strain fields (\#8-\#9). Taking grain averages of the latter (\#10\#11), a correlation analysis (shown in \#12 for one elastic strain component) can be carried out to compare with the corresponding synthetic data (\#5), component by component of stress and elastic strain.

Figure 2 shows the correlation between the six independent grain-averaged stress components from the synthetic experiment and the corresponding instantiations based on Eshelby's approximation. Similar but not identical-due the elastic anisotropy of the $\mathrm{Cu}$ single crystals-correlation plots (not shown) were obtained for the elastic strain components. The clouds are made of points obtained for each of the 14592 grains in the microstructure. The linear regression line is shown in red and, for reference, the $\mathrm{y}=\mathrm{x}$ line is shown in black. The slopes (to be denoted $\mathrm{m}_{\mathrm{ij}}^{\varepsilon}$ and $\mathrm{m}_{\mathrm{ij}}^{\sigma}$, respectively), y-intercepts and $\mathrm{R}^{2}$ values of the linear regression lines for the elastic micro-strains $\left(\times 10^{-6}\right)$ and stresses (MPa) are reported in Table 1. While slopes of 1 would represent a good match, on average, of the experimental and instantiated fields, the obtained higher-than-one slopes clearly show that the absolute values of the instantiated elastic strain and stress components are over-predicted, on average, using Eshelby's approximation. The reasons for this imperfect correlation are various. Under this approximation, eigenstrains are estimated assuming that the individual grains are spherical and embedded in a homogeneous infinite medium. However, the actual spatial heterogeneity, which determines stronger elastic interactions of grains with their nearest neighbors, introduces deviations from the homogeneous matrix assumption. In addition, while by virtue of Voronoi's construction, the shape of the grains is equiaxed on average, individual grains can deviate from this average shape introducing additional deviations from Eshelby's approximation. Furthermore, the finite size and specific geometry of the specimen (cylindrical in this case) differs from the assumption of an infinite homogenous matrix that constraints the elastic behavior of each grain, and therefore can also have a deleterious effect on the correlation between the predicted and measured fields.

The deviation of the slopes of the regression lines with respect 45 degrees (i.e. deviation of $\mathrm{m}_{\mathrm{ij}}^{\varepsilon}$ and $\mathrm{m}_{\mathrm{ij}}^{\sigma}$ from 1) in the above grain-by-grain correlations can be used to calibrate the $\beta_{\mathrm{ij}}$ matrix 
proposed in Eq. (3) to correct the eigenstrain field, for a better matching with the data. Defining $\beta_{\mathrm{ij}}=2 /\left(\mathrm{m}_{\mathrm{ij}}^{\varepsilon}+\mathrm{m}_{\mathrm{ij}}^{\sigma}\right)$ we obtain:

$\beta_{\mathrm{ij}}=\left[\begin{array}{lll}0.63 & 0.68 & 0.67 \\ & 0.63 & 0.66 \\ & & 0.61\end{array}\right]$

Figure 3 shows the correlation plots for the six independent grain-averaged stress components from the synthetic experiments and the corresponding instantiations, after rescaling the eigenstrains using Eq. (3) with the numerical values of $\beta_{\mathrm{ij}}$ given in Eq. (4). The slopes for all six components of stress (and also of elastic strain, not shown) are very close to 1, indicating that, on average, the instantiation is in good agreement with the data. The slopes, $y$-intercepts and $\mathrm{R}^{2}$ values of the regression lines for the elastic micro-strain and stress components are reported in Table 2. Using the eigenstrain field resulting from Eqs. (3-4) (\#13 in Fig. 1), the measured RS can be accounted for in subsequent TEVPFFT calculations. For example, plot \#14 in Fig. 1 shows the tensile stress-strain curves for the cases without consideration of RS (same as \#2), and with RS obtained with the proposed approximation. The comparison shows the effect of RS, which modify the shape of the macroscopic stress-strain curve, especially in the elastoplastic transition. In this particular case, the RS tend to delay the onset of plasticity, by leaving plastically soft grains in compression after unloading, a local state that needs to be overcome upon reloading the polycrystal in tension.

\section{4- Conclusions}

In this letter we have demonstrated a methodology to incorporate 3-D measurements of grainaveraged RS for instantiation of image-based full-field CP simulations. The workflow, illustrated in Fig.1, requires performing several operations on orientation fields and grain-averaged data, which, in the context of a 3-D experiment, can be obtained by ff-HEDM or concurrent nf- and ffHEDM. This sequence of operations can be automated for rapid and convenient instantiation of CP simulations.

The key element of the proposed methodology is the calibration of a set of eigenstrains in the grains to reproduce, after equilibration, the measured RS. The starting point is Eshelby's approximation, which in turn is corrected using information on the slopes of the regression lines 
resulting from the imperfect correlation between measured and instantiated grain-averaged field components. The slopes and y-intercepts after this refinement are close to 1 and 0 , respectively. For even better matching, further correction could be performed through a narrow 6-dimensional search of the independent components of $\beta_{\mathrm{ij}}$ centered on the identified values using the regression slopes. This onerous further correction of Eshelby's approximation, however, may be well within the experimental error associated with the determination of the grain-averaged residual stresses [21] and therefore its potential benefit should be judiciously considered. An alternative method, which improves upon the Eshelby-based model presented here, can be formulated as the problem of determining a set of eigenstrains in each grain of the actual microstructure, such that the grain-averaged elastic strains obtained from a thermo-elastic calculation are in agreement with the measured ones. The eigenstrain calibration can be expressed as the minimization of a cost function representing the difference between the measured and predicted grain's elastic strains [22]. This method is under investigation and will be reported elsewhere [23].

The added capability to image-based CP models to deliver accurate numerical simulations with direct input from microstructural data collected in existing light sources may be instrumental to fully reduce 3-D data collected, e.g. under dynamic deformation conditions, in future instruments and/or future light sources. Under dynamic conditions, a much limited number of noise-prone diffraction views of the sample will be available for data reduction, from diffraction images to 3$\mathrm{D}$ microstructural images. The availability of models that can accurately predict the evolution of microstates starting from very carefully pre-characterized samples may help constraining 3-D reconstruction under such stringent experimental conditions.

\section{Acknowledgements}

This work was supported by Los Alamos National Laboratory's Laboratory-Directed Research and Development (LDRD) program. Fruitful discussions with Cristina Garcia-Cardona, Marian Anghel (LANL), Jette Oddershede, Grethe Winther (DTU) and Tony Rollett (CMU) are acknowledged. 


\section{References}

[1] U. Lienert, S.F. Li, C.M. Hefferan, J. Lind, R.M. Suter, J.V. Bernier, N.R. Barton, M.C. Brandes, M.J. Mills, M.P. Miller, B. Jakobsen, W. Pantleon, JOM 63 (2011) 70.

[2] R. Dingreville, R.A Karnesky, G. Puel, J.H. Schmitt, J. Mat. Sci. 5 (2016) 1178-1203.

[3] H Wang, B Clausen, L Capolungo, I.J. Beyerlein, J. Wang, C.N. Tomé, Int. J. Plast. (2016) 79, 275-292.

[4] H.F. Poulsen, Three-dimensional X-ray diffraction microscopy: mapping polycrystals and their dynamics, Springer, Berlin, 2004.

[5] R.M. Suter, D. Hennessy, C. Xiao, U. Lienert, Rev. Sci. Instr. 77 (2006) 123905.

[6] G. Johnson, A. King, M. Goncalves Honnicke, J. Marrow, W. Ludwig, J. Appl. Cryst. 41 (2008) 310-318.

[7] R. Pokharel, J. Lind, A.K Kanjarla, R.A. Lebensohn, S.F. Li, P. Kenesei, R.M. Suter, A.D. Rollett, Ann. Rev. Cond. Mat. Phys. 5 (2014) 317-346.

[8] R. Pokharel, J. Lind, S.F. Li, P. Kenesei, R.A. Lebensohn, R.M. Suter, A.D. Rollett, Int. J. Plast. 67 (2015) 217-234.

[9] J. Oddershede, S. Schmidt, H.F. Poulsen, L. Margulies, J. Wright, M. Moscicki, W. Reimers, G. Winther, Mat. Charact. (2011) 62, 651.

[10] J.V. Bernier, N.R. Barton, U. Lienert, M.P. Miller, J. Strain Anal. Eng. 46 (2011) 527-547.

[11] E.J Lieberman, R.A Lebensohn, D.B. Menasche, C.A. Bronkhorst, A.D Rollett, Acta Mater. 116 (2016) 270-280.

[12] A. Cerrone, C. Stein, R. Pokharel, C. Hefferan, J. Lind, H. Tucker, R. Suter, A. Rollett . A. Ingraffea, Mod. Sim. Mat. Sci. Eng. 23 (2015) 035006.

[13] R.A. Lebensohn, A.K. Kanjarla, P. Eisenlohr, Int. J. Plast. 32-33 (2012) 59-69.

[14] B.S. Anglin, R.A. Lebensohn, A.D. Rollett, Comp. Mat. Sci. 87 (2014) 209-217.

[15] T.J Turner, P.A. Shade, J.V. Bernier, S.F. Li, J.C. Schuren, J. Lind, U. Lienert, P. Kenesei, R.M. Suter, B. Blank, J. Almer, Integr. Mater. Manuf. Innov. 5 (2016) 1-9. 
[16] T.J Turner, P.A. Shade, J.V. Bernier, S.F. Li, J.C. Schuren, J. Lind, U. Lienert, P. Kenesei, R.M. Suter, J. Almer, Metall. Mater. Trans. A, in press (doi:10.1007/s11661-016-3868-x).

[17] R. Pokharel et al., in preparation.

[18] T. Mura, Micromechanics of Defects in Solids, Martinus-Nijhoff, Dordrecht, 1987.

[19] A.V. Hershey, ASME J. Appl. Mech. 21 (1954), 236-240.

[20] A. Lyckegaard, E.M. Lauridsen, W. Ludwig, R.W. Fonda, H.F. Poulsen, Adv. Eng. Mat. 13 (2011), 165-170.

[21] J. Oddershede, S. Schmidt, H.F. Poulsen, H. Sørensen, J. Wright, W. Reimers, J. Appl. Cryst. 43 (2010) 539-49.

[22] C. Garcia-Cardona, R.A. Lebensohn, M. Anghel, Int. J. Num. Meth. Eng., submitted.

[23] C. Garcia-Cardona, R. Pokharel, R.A. Lebensohn, in preparation. 
Tables

\begin{tabular}{|l|c|c|c|c|c|c|c|c|c|c|c|c|}
\hline component & $\varepsilon_{11}^{\mathrm{e}}$ & $\varepsilon_{22}^{\mathrm{e}}$ & $\varepsilon_{33}^{\mathrm{e}}$ & $\varepsilon_{23}^{\mathrm{e}}$ & $\varepsilon_{31}^{\mathrm{e}}$ & $\varepsilon_{12}^{\mathrm{e}}$ & $\sigma_{11}$ & $\sigma_{22}$ & $\sigma_{33}$ & $\sigma_{23}$ & $\sigma_{31}$ & $\sigma_{12}$ \\
\hline $\begin{array}{l}\text { slope } \\
\left(\mathrm{m}_{\mathrm{ij}}^{\varepsilon} / \mathrm{m}_{\mathrm{ij}}^{\sigma}\right)\end{array}$ & 1.51 & 1.51 & 1.55 & 1.54 & 1.53 & 1.50 & 1.65 & 1.65 & 1.71 & 1.48 & 1.46 & 1.44 \\
\hline $\begin{array}{l}\mathrm{y} \text {-intercept } \\
{\left[\mathrm{x} 10^{-6} / \mathrm{MPa}\right]}\end{array}$ & -0.93 & 1.19 & 3.55 & -0.14 & 0.08 & -0.02 & 0.02 & -0.01 & 0.70 & -0.01 & 0.01 & -0.01 \\
\hline $\mathrm{R}^{2}$ & 0.95 & 0.95 & 0.95 & 0.93 & 0.93 & 0.94 & 0.92 & 0.91 & 0.94 & 0.89 & 0.88 & 0.88 \\
\hline
\end{tabular}

Table 1: Slopes, $\mathrm{y}$-intercepts and $\mathrm{R}^{2}$-values of the linear regression lines for the correlation between the grain-averaged residual elastic micro-strain and stress components from synthetic experiments and the corresponding instantiations based on Eshelby's approximation.

\begin{tabular}{|l|c|c|c|c|c|c|c|c|c|c|c|c|}
\hline component & $\varepsilon_{11}^{\mathrm{e}}$ & $\varepsilon_{22}^{\mathrm{e}}$ & $\varepsilon_{33}^{\mathrm{e}}$ & $\varepsilon_{23}^{\mathrm{e}}$ & $\varepsilon_{31}^{\mathrm{e}}$ & $\varepsilon_{12}^{\mathrm{e}}$ & $\sigma_{11}$ & $\sigma_{22}$ & $\sigma_{33}$ & $\sigma_{23}$ & $\sigma_{31}$ & $\sigma_{12}$ \\
\hline $\begin{array}{l}\text { slope } \\
\left(\mathrm{m}_{\mathrm{ij}}^{\varepsilon} / \mathrm{m}_{\mathrm{ij}}^{\sigma}\right)\end{array}$ & 0.97 & 0.97 & 0.96 & 1.01 & 1.01 & 1.01 & 1.06 & 1.06 & 1.06 & 0.98 & 0.97 & 0.97 \\
\hline $\begin{array}{l}\mathrm{y}-\mathrm{intercept} \\
{\left[\mathrm{x} 10^{-6} / \mathrm{MPa}\right]}\end{array}$ & -0.36 & 0.50 & 2.06 & -0.08 & 0.05 & -0.02 & 0.01 & -0.00 & 0.46 & -0.01 & 0.01 & -0.01 \\
\hline $\mathrm{R}^{2}$ & 0.95 & 0.95 & 0.96 & 0.95 & 0.95 & 0.96 & 0.92 & 0.91 & 0.94 & 0.92 & 0.92 & 0.92 \\
\hline
\end{tabular}

Table 2: Slopes, $\mathrm{y}$-intercepts and $\mathrm{R}^{2}$-values of the linear regression lines for the correlation between the grain-averaged residual elastic micro-strain and stress components from synthetic experiments and the corresponding instantiations based on Eshelby's approximation refinement, after rescaling the eigenstrains using Eq. (3) with the numerical values of $\beta_{\mathrm{ij}}$ given in Eq. (4). 

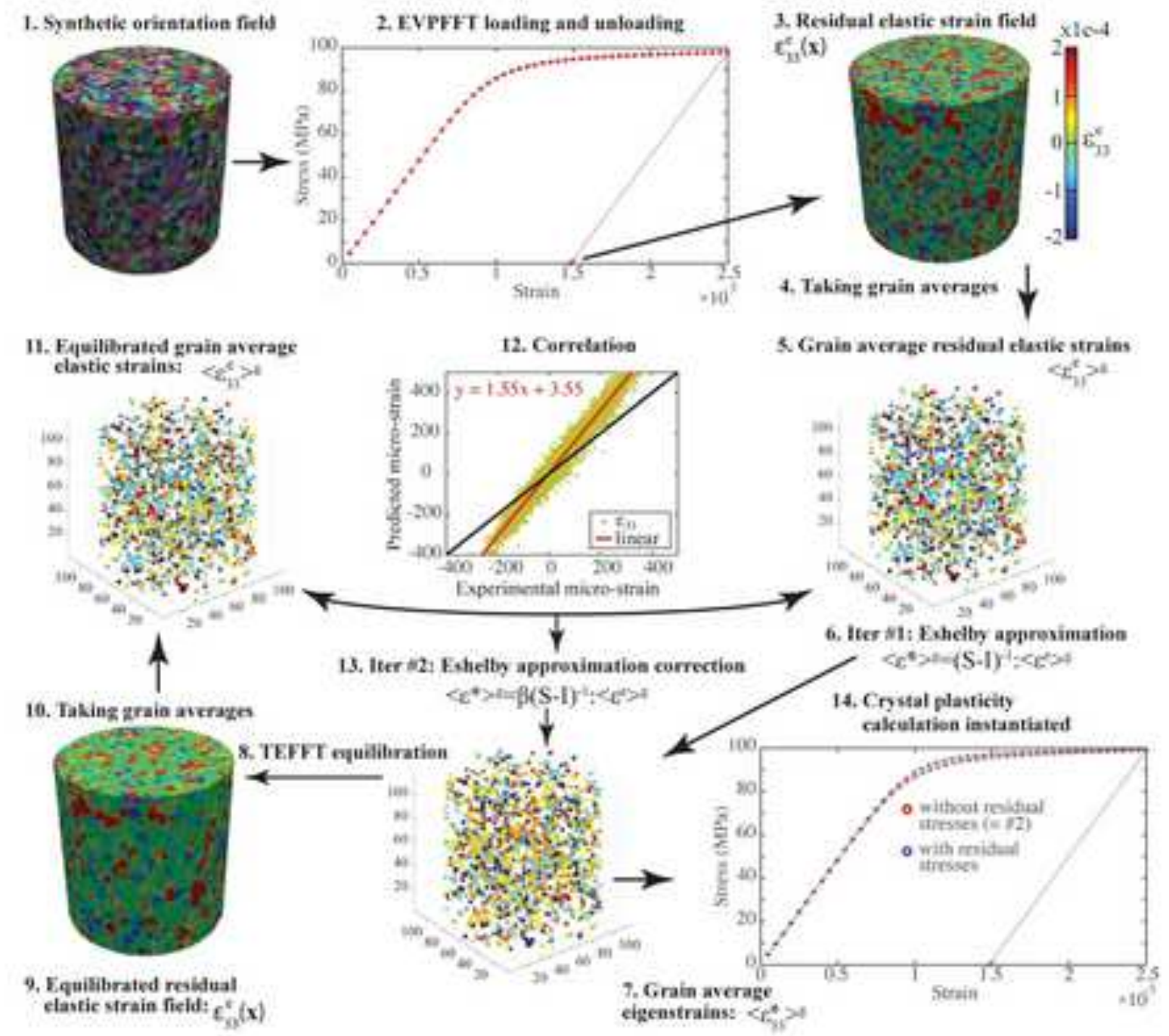

Figure 1: Schematic of proposed refinement of Eshelby's approximation. Continuous 3-D plots (\#1, \#3 and $\# 9$ ) represent field data, while discrete sphere plots ( $\# 5, \# 7$ and \#11) represent grain averaged data (sphere centers $=$ center of mass of grains, color $=$ constant average value of corresponding field in the grain). 

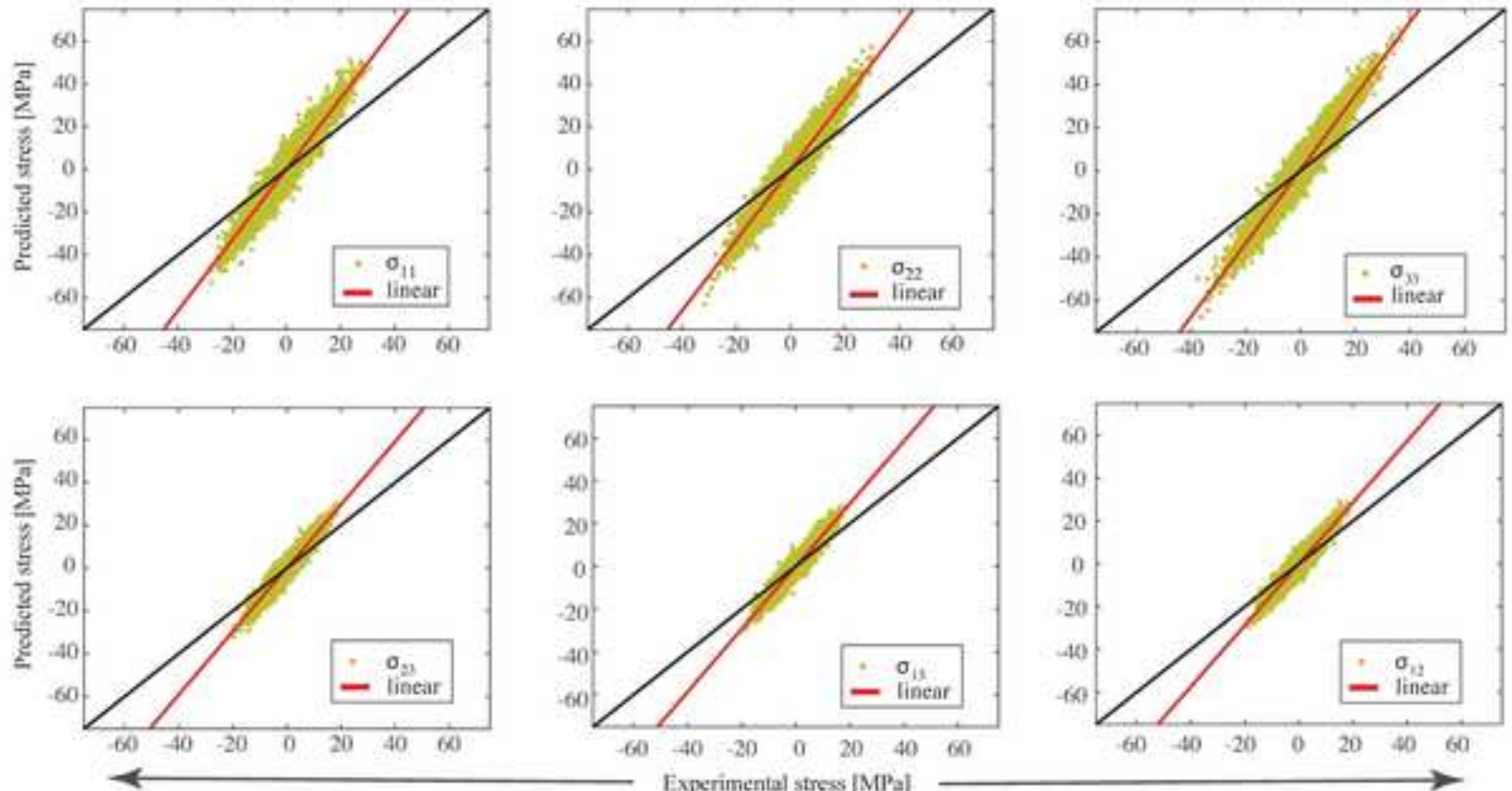

Figure 2: Correlation between the six independent grain-averaged stress components from the synthetic experiment and the corresponding instantiations based on Eshelby's approximation. 

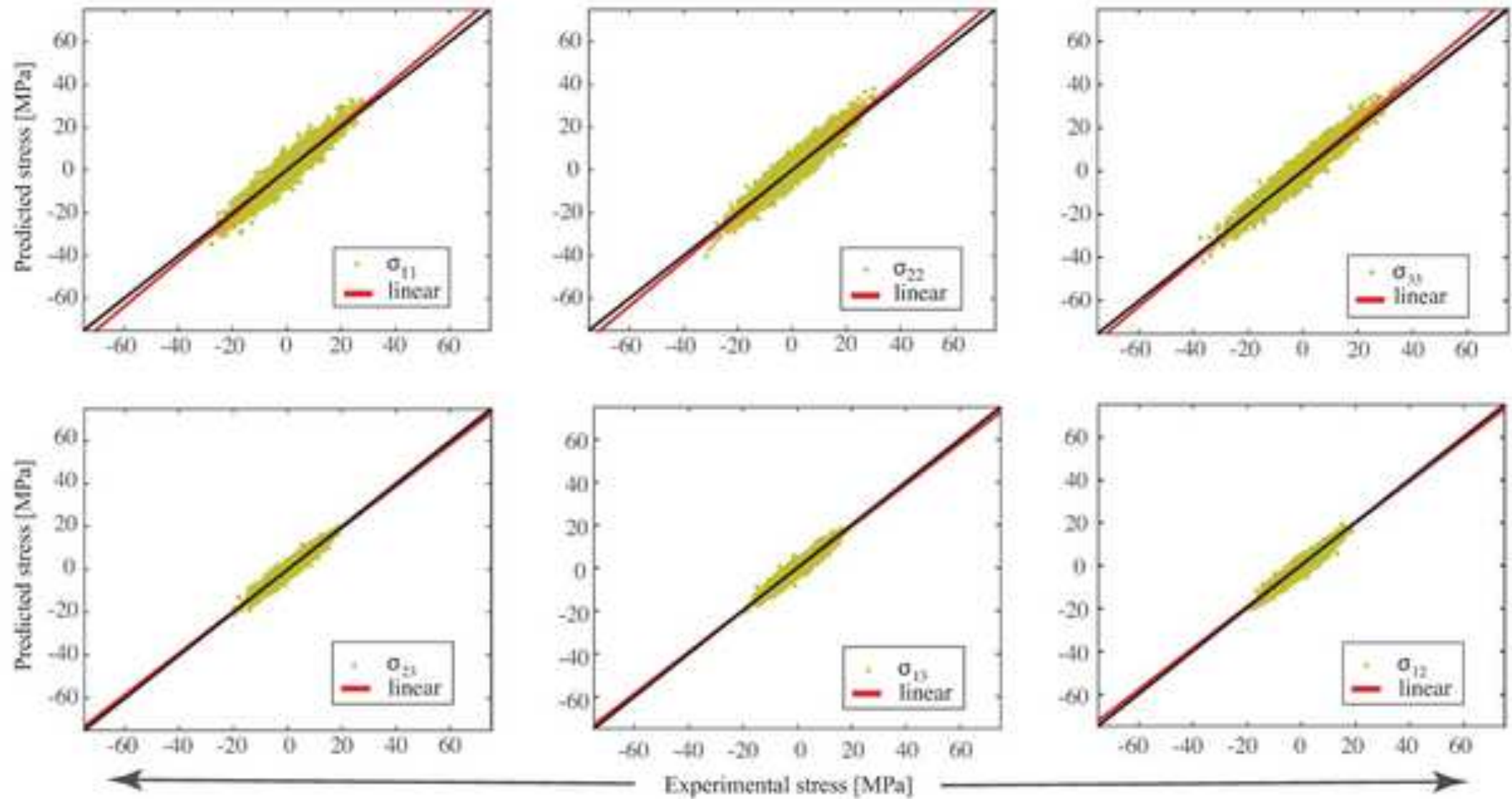

Figure 3: Correlation between the six independent grain-averaged stress components from the synthetic experiments and corresponding instantiations, after rescaling the eigenstrains using Eq. (3) with the numerical values of $\beta_{\mathrm{ij}}$ given in Eq. (4). 
1. Synthetic orientation field

2. EVPFFT loading and unloading

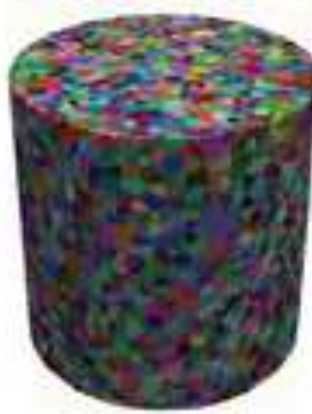

11. Equilibrated grain average elastic strains: $\left\langle\varepsilon_{33}^{e}\right\rangle 8$
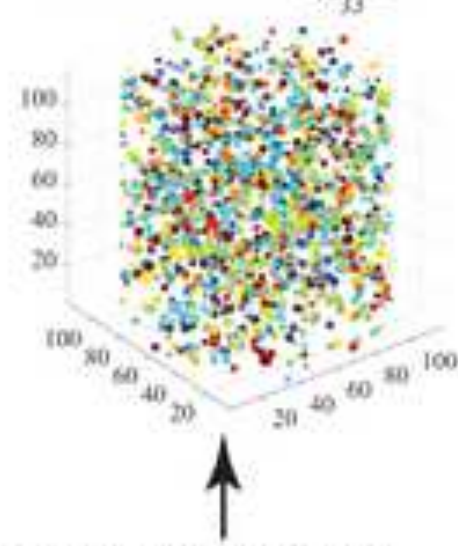

10. Taking grain averages

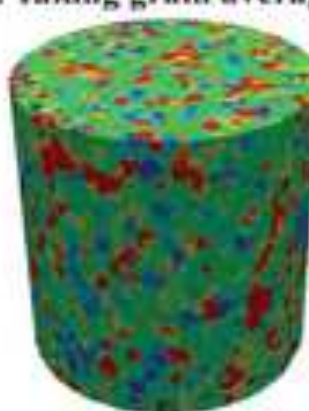

9. Equilibrated residual elastic strain field: $\varepsilon_{33}^{c}(\mathbf{x})$
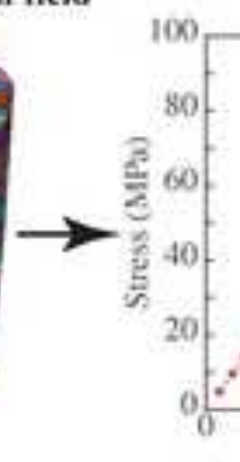

2. EVPFFT loading and unloading

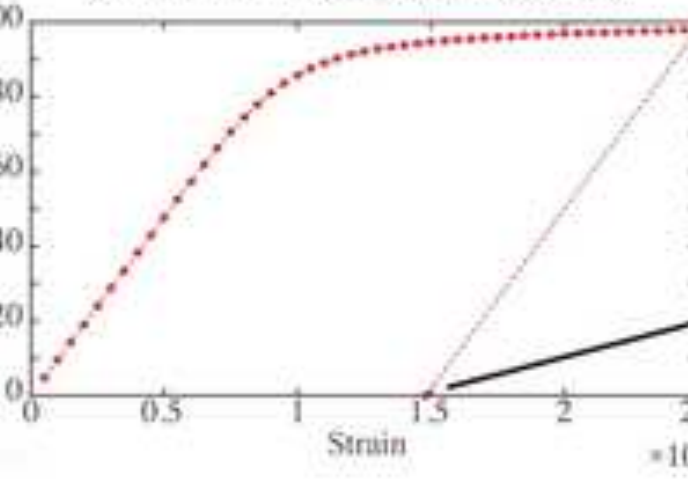

12. Correlation

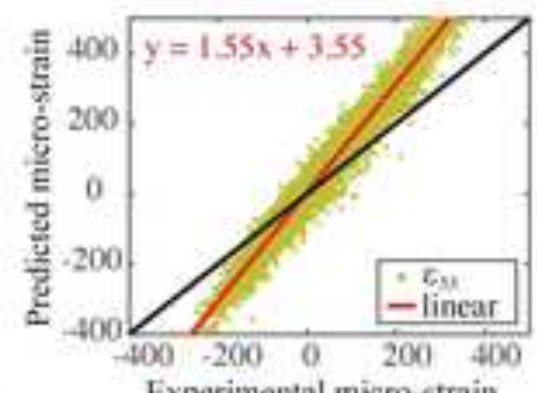

Experimental micro-strain

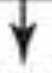

\section{.}

8. TEFFT equilibration

$\left\langle\varepsilon^{*}>\theta-\beta(S-I)^{-1}:\left\langle\Omega^{2}>8\right.\right.$

13. Iter 12 : Eshelby approximation correction

$\left.i^{\prime}-1\right)^{2}:<\varepsilon^{2}>x$

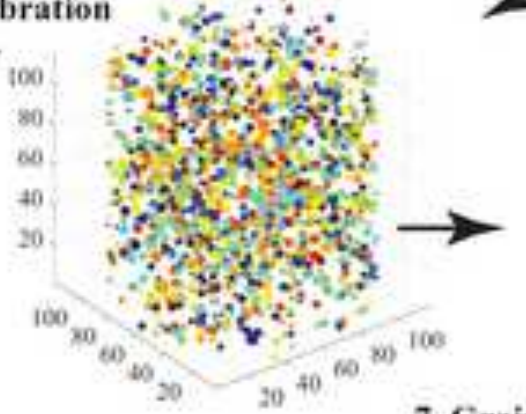

3. Residual elastic strain field

$\varepsilon_{33}^{e}(\mathbf{x})$
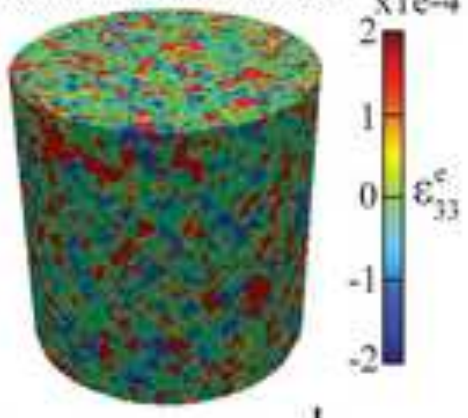

4. Taking grain averages $\downarrow$

5. Grain average residual elastic strains

$$
\left\langle\varepsilon_{33}^{e}>8\right.
$$
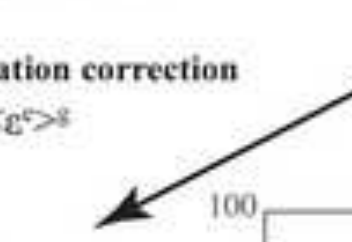

\section{$100 \quad$ calculation instantiated \\ 14. Crystal plasticity}

$<\varepsilon^{*}>\sin (\mathrm{S}-1)^{-1}:<\varepsilon>5$

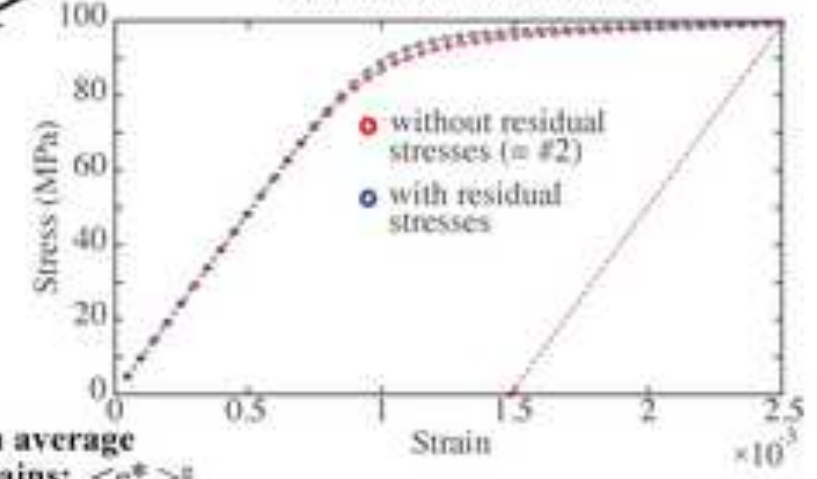

7. Grain average eigenstrains: $\left\langle\varepsilon_{23}^{*}\right\rangle$ 